\title{
La canine et l'enfant
}

\section{RÉSUMÉ}

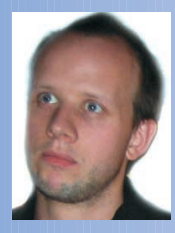

Mathieu A. DERBANNE

Ancien AHU,

Service d'odontologie du Pr Bruno Gogly,

Groupe Hospitalier

Albert Chenevier - Henri Mondor,

AP-HP,

Créteil.

\section{Marguerite-M arie LANDRU}

MCU-PH,

Service d'odontologie du Pr Bruno Gogly,

Groupe Hospitalier

Albert Chenevier - Henri Mondor

AP-HP,

Créteil.

Les canines temporaires et permanentes jouent un rôle majeur dans les phénomènes de croissance des arcades dentaires. La mise en place et l'établissement d'une fonction correcte des canines temporaires permettront de prévenir l'apparition de dysmorphoses lors des stades de dentures ultérieurs. Il est ainsi capital de surveiller et de préserver ces canines. L'évolution de la canine permanente représente aussi un moment clé des phénomènes de dentition. L'observation et la surveillance précoce de son évolution sont les garants de son évolution correcte en permettant d'intercepter les inclusions ou les malpositions sévères et préjudiciables non seulement à la fonction mais aussi à l'esthétique dentaire de l'adolescent et de l'adulte. A chaque étape de dentition, les canines, temporaires ou permanentes font l'objet de soins particuliers : denture temporaire, denture mixte et denture adolescente.

- canine

- enfant

- croissance

- dent temporaire

- dent permanente 


\section{Introduction}

$>$

L'importance des dents temporaires est parfois sous estimée eu égard à leur caractère temporaire et à leur physiologie particulière qui les mène à l'exfoliation. Les dents temporaires jouent un rôle majeur dans le maintien de la dimension des formes et de la taille des arcades dentaires, dans I'harmonie de la croissance faciale. La canine temporaire et la canine permanente ont un rôle de véritable clé de voute des arcades dentaires. La préservation de la canine temporaire et la mise en place sur l'arcade de la canine permanente feront partie intégrante des objectifs de nos plans de traitement et du suivi buccodentaire des enfants.

\section{La canine temporaire}

Comme l'ensemble des dents temporaires, la canine temporaire débute son évolution intra utero pour faire son évolution autour de l'âge de 24 mois, après les incisives et les premières molaires temporaires. La chronologie de son évolution est reprise dans le tableau I d'après Lautrou[1].

\section{Tableau I}

\begin{tabular}{lccccc}
\hline & I & II & III & IV & V \\
\hline $\begin{array}{l}\text { Début de minéralisation } \\
\text { coronaire }\end{array}$ & 4 mois IU1 & 4 mois IU & 5 mois IU & 5 mois IU & 6 mois IU \\
\hline Couronne achevée & 3 mois & 3 mois & 9 mois & 6 mois & 12 mois IU \\
\hline Éruption & 6 mois & 12 mois & 24 mois & 18 mois & 30 mois IU \\
\hline $\begin{array}{l}\text { Édification radiculaire } \\
\text { complète }\end{array}$ & 2 ans & 2,5 ans & 3 ans & 3 ans & 4 ans \\
\hline Début de résorption & 4 ans & 5 ans & 8 ans & 6 ans & 7 ans \\
\hline Chute & 7 ans & 8 ans & $10-11$ ans & $9-10$ ans & $10-11$ ans \\
\hline
\end{tabular}

II existe une certaine variabilité dans le développement occlusal de l'enfant. Néanmoins trois éléments sont indicateurs d'un développement correct: la présence de diastèmes interincisifs[2], l'existence de diastèmes simiens, en mésial de la canine temporaire maxillaire et en distal de la canine temporaire mandibulaire[3], et un plan de Chapman droit ou à marche mésiale[4]. Avant la phase de denture mixte, la croissance transversale des maxillaires est relativement faible, la largeur de l'arcade étant déterminée par la position des canines.

II n'est pas rare d'observer des pointes canines totalement abrasée lorsque l'âge d'exfoliation de la canine s'approche (aux alentours de 10 ans

\footnotetext{
1 Intra Utérin.
} 


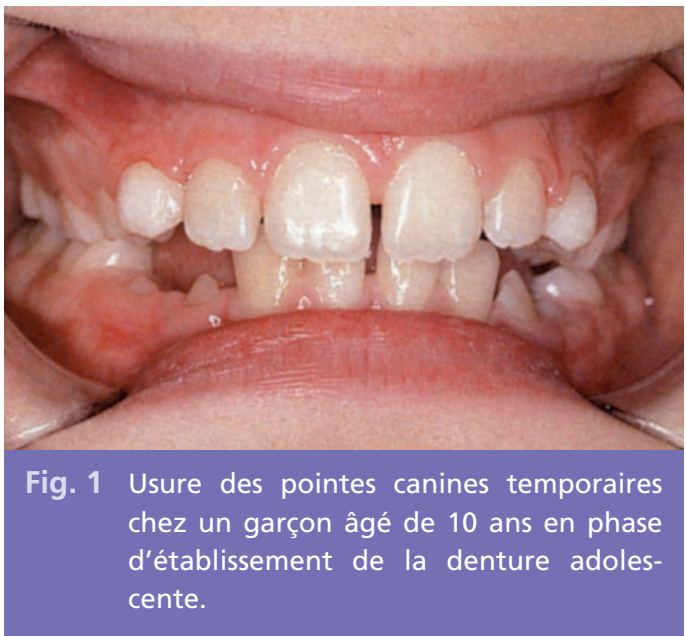

- fig. 1). Cette usure est normale et découle de la fonction masticatoire de l'enfant mais aussi des phénomènes de bruxisme, très fréquents et sans gravité lorsqu'ils restent modérés chez l'enfant[5]. Dans le cas contraire, I'absence d'abrasion doit nous faire suspecter l'existence d'une malocclusion que l'examen clinique nous permettra de déceler.

En denture temporaire et en denture mixte, la canine peut être impliquée dans le développement d'anomalies du sens transversal et du sens sagittal. Dans le sens transversal, il n'est pas rare d'observer des inversions de l'occlusion unilatérales ou bilatérales. Une cause fonctionnelle doit être recherchée en de pareils cas. Fréquemment, une anomalie de la posture ou de la fonction linguale est associée à ce type de malocclusion, la langue étant, d'après Deffez, "le conformateur de la cavité buccale»[6,7]. Une position de langue basse au repos, influant négativement sur la croissance transversale du maxillaire, entraîne une endoalvéolie maxillaire uni ou bilatérale en fonction du guide canin et de la cinétique mandibulaire. Le développement de dysfonctions linguales est favorisé par l'existence d'habitudes néfastes comme une succion digitale ou l'usage d'une tétine qui engendrent sou-

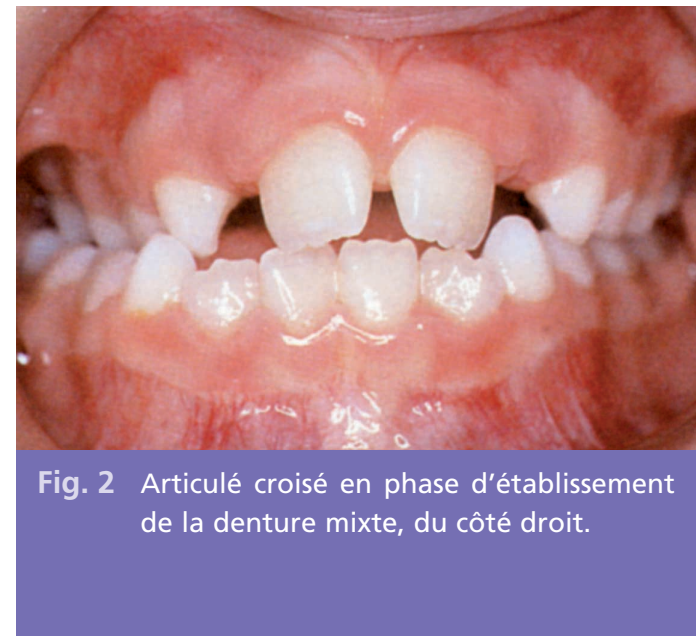

vent une persistance de la déglutition primaire[8].

Le traitement précoce de ce type d'anomalie est souhaitable de façon à éviter la pérennisation de la position anormale de la mandibule entraînée par une anomalie unilatérale ou le blocage de la croissance transversale maxillaire dans le cas d'anomalies bilatérales; la croissance osseuse étant, certes sous la dépendance de facteurs génétiques, mais aussi de facteurs environnementaux et fonctionnels. Le traitement de ces anomalies est réalisé par appareillage amovible. L'appareillage, de type plaque de Hawley consiste à déverrouiller l'occlusion par des plans de surélévation et à stimuler la croissance suturale des maxillaires à I'aide d'un vérin médian placé sur la plaque. Concomitamment au rétablissement $d$ 'une largeur maxillaire correcte, les pointes canines devront être meulées afin de lever toute interférence qui induirait une récidive après la fin du traitement orthopédique (fig. 2).

Les canines temporaires ont un rôle déterminant sur la croissance du maxillaire. À ce titre, la conservation des canines temporaires est un objectif majeur des traitements qui pourront être entrepris en présence de pathologies carieuses. Les canines temporaires doivent donc 

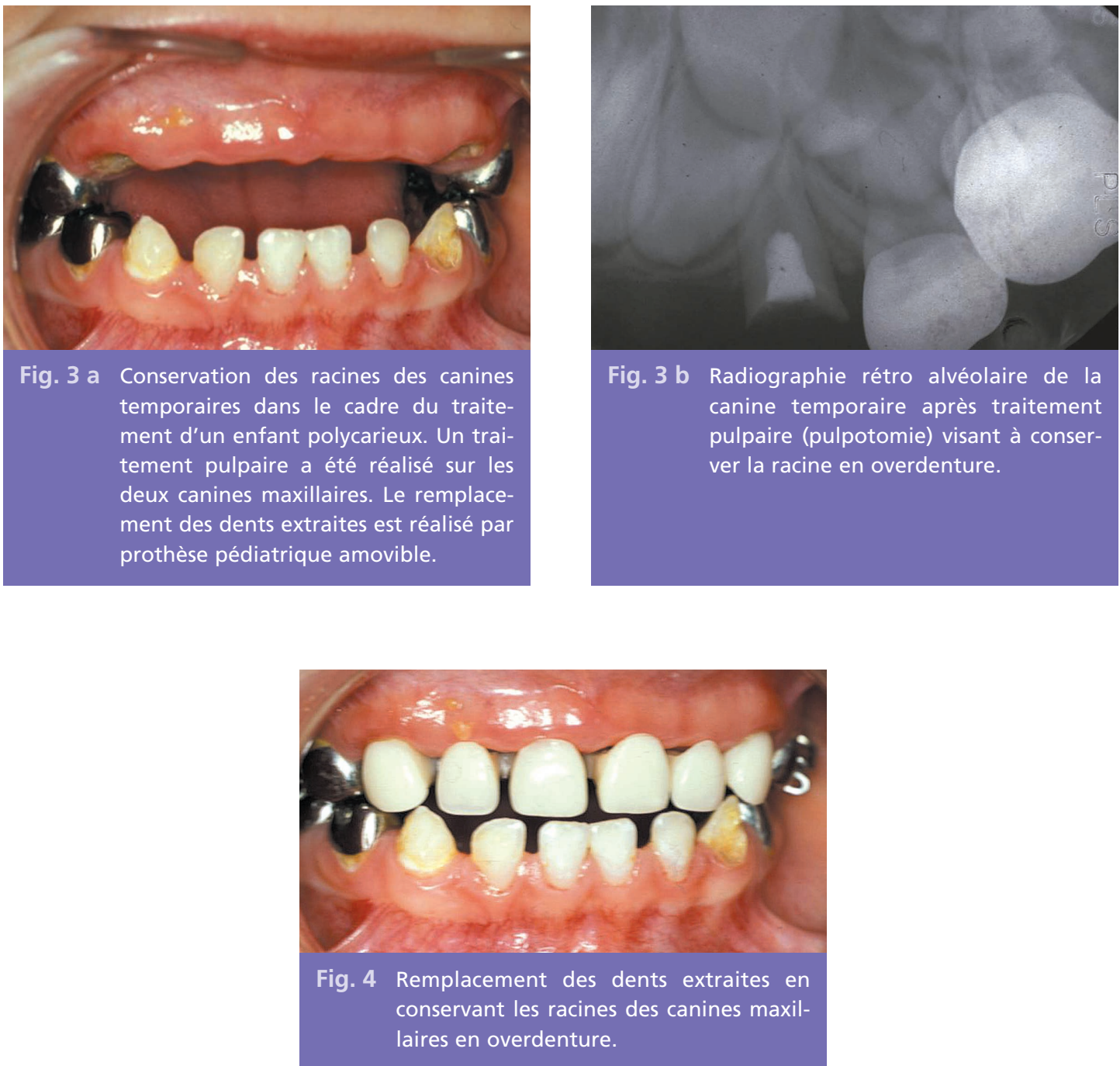

être maintenues sur l'arcade jusqu'à l'éruption des incisives permanentes. L'extraction précoce d'une canine temporaire entraîne la migration mésiale des dents qui lui sont immédiatement distales, en réduisant l'espace nécessaire à l'éruption des canines permanentes. On observe par ailleurs, une migration des incisives en direction de la canine extraite, causant une modification de la position du point inter incisif, en particulier lorsque les extractions interviennent avant la fin de la constitution de la denture mixte vers 10 ans[9].
Lors de la réalisation de traitements conservateurs, en particulier chez les patients polycarieux, des extractions sont souvent inévitables. Nous chercherons néanmoins à maintenir les canines temporaires. Il est possible, dans les cas d'atteintes carieuses sévères de ne conserver que leurs racines. Le remplacement des dents extraites et de la couronne des canines temporaires étant alors réalisé en appui sur les racines résiduelles qui jouent alors leur rôle de maintien de la largeur d'arcade (fig. 3 a et $b$ et fig. 4). 


\section{En denture mixte}

Pendant la phase d'établissement de la denture mixte, la question de l'extraction des canines temporaires peut se poser face un encombrement dans le secteur incisif. II est nécessaire de prendre en compte l'asynchronisme entre la croissance osseuse et l'évolution des dents permanentes, la première étant souvent moins précoce que la seconde. Ainsi, un encombrement modéré à la mandibule du secteur incisif se résout souvent de lui-même lorsque la largeur intercanine augmente sous l'effet de la croissance osseuse (fig. 5). La largeur intercanine atteint son maximum lorsque les incisives permanentes ont totalement terminé leur éruption[4]. L'extraction des canines temporaires mandibulaires face à un encombrement incisif modéré pendant la

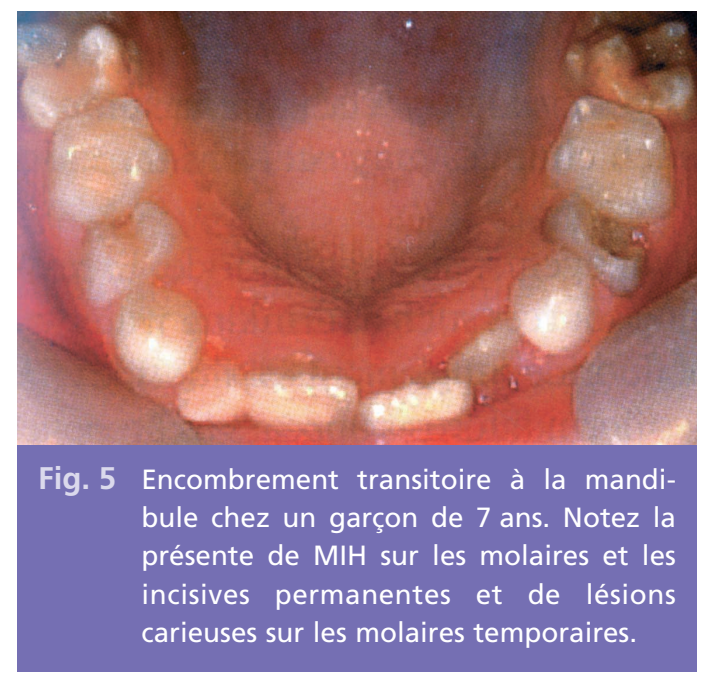

phase de constitution de la denture mixte ne doit pas être menée de façon trop précoce : il faut attendre la croissance osseuse[4].

\section{La canine permanente}

D'après Lautrou, les canines permanentes évoluent vers l'âge de 10 ans à la mandibule, et 11 ans au maxillaire ; les détails de leur évolution sont repris dans le tableau II[1].

\section{Tableau II}

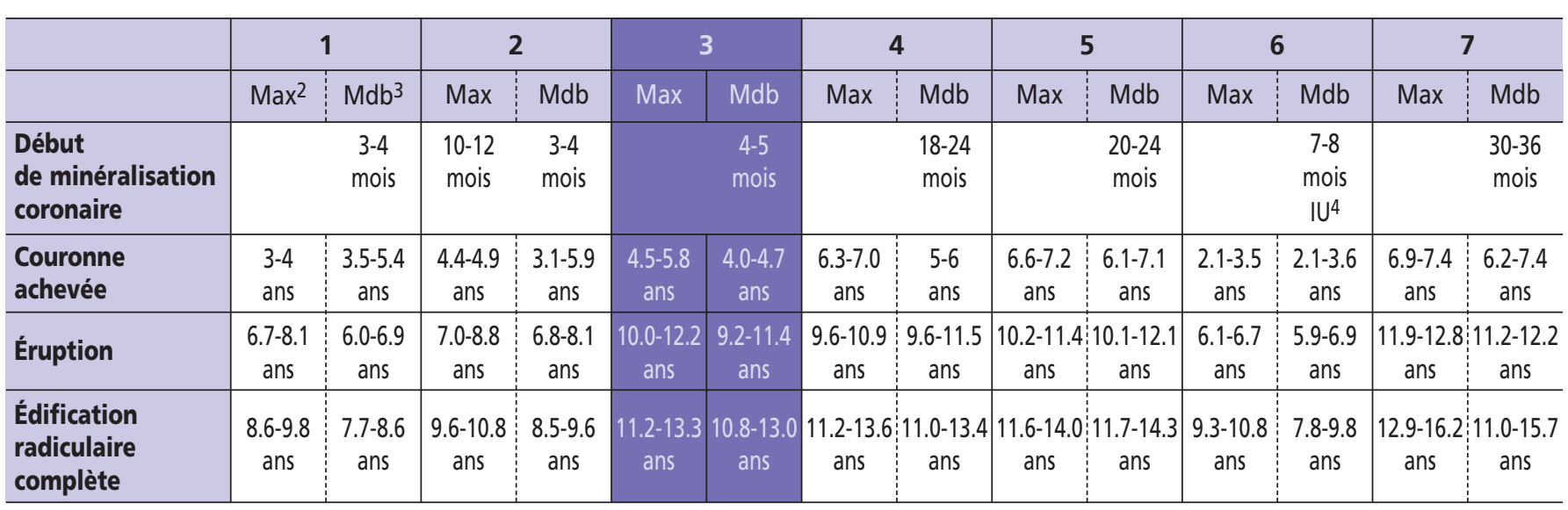

2 Maxillaire. ${ }^{3}$ Mandibule. ${ }^{4}$ Intra Utérin. 
Le chemin d'éruption de dents permanentes peut être perturbé, aboutissant parfois à son inclusion. La dent la plus fréquemment incluse est la troisième molaire, suivie par la canine maxillaire permanente. Une éruption ectopique de la canine maxillaire se produit chez $2 \%$ de la population. Dans $85 \%$ des cas d'inclusion, la canine se situe en position palatine[10]. Le risque d'inclusion de la canine permanente maxillaire est majoré quand l'incisive latérale permanente adjacente est absente ou dysmorphique, la racine de l'incisive latérale servant de guide à l'éruption de la canine (fig. 6).

Il est important de déceler les signes d'une évolution anormale de la canine permanente de manière à prévenir son inclusion d'une part, et des phénomènes de résorption de la racine de I'incisive latérale permanente d'autre part qui se produisent dans $12 \%$ des cas de canines permanentes en voie d'inclusion.

Le chemin normal d'éruption de la canine maxillaire est légèrement vestibulé par rapport à l'arcade dentaire. À l'âge de 10 ans, la palpation de la canine maxillaire au niveau du vestibule est normale. Sinon, on doit suspecter la possibilité d'une canine permanente en position anormale et réaliser les examens complémentaires nécessaires. Quand les canines perma- nentes sont en position vestibulaires, leur chemin d'éruption sera vertical et l'éruption en position vestibulaire haute, parfois au dessus de la ligne mucogingivale. Dans les cas de position palatine, le risque d'inclusion et de résorption est majoré. Un diagnostic précoce et une intervention précoce sont alors souhaitables[11].

Un autre signe, plus précoce doit être recherché. Les canines utilisant les incisives latérales comme guide d'éruption, une position anormale de la canine permanente peut engendrer une rotation disto vestibulaire de l'incisive latérale[12]. Cette position des incisives latérales indique souvent des canines maxillaires dont l'axe, au lieu d'être vertical, est basculé. Le contrôle radiographique sur radiogramme panoramique permet de confirmer notre hypothèse clinique (fig. 7 et 8).

Enfin, une asymétrie importante dans la cinétique d'éruption des canines permanentes doit faire suspecter une possibilité d'inclusion de la canine, l'examen clinique (palpation des vestibules) et radiographique (panoramique) est alors indiqué pour confirmer ou infirmer notre hypothèse.

En fin de phase de denture mixte, si une canine permanente est en position anormale, l'extraction de la canine temporaire correspon-

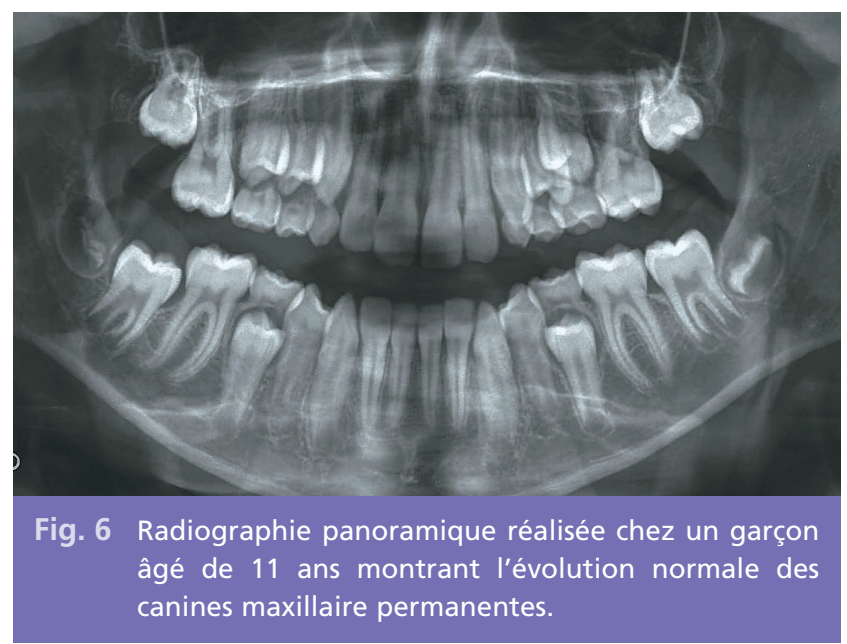




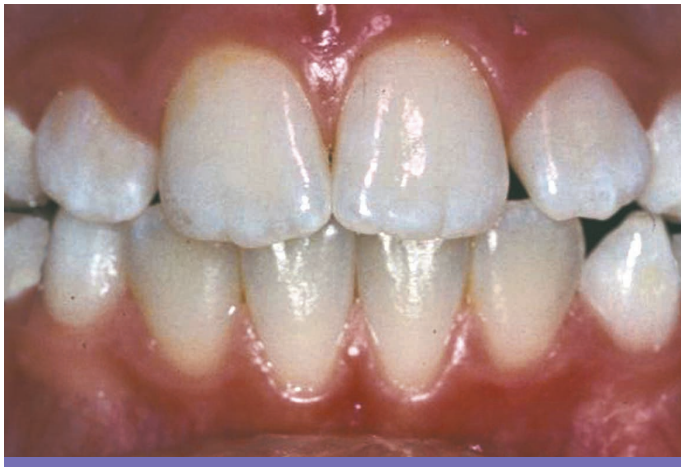

Fig. 7 Rotation vestibulo-distale des incisives latérales maxillaires chez un garçon âgé de 10 ans. Cette position des latérales doit faire suspecter une malposition des canines permanentes.

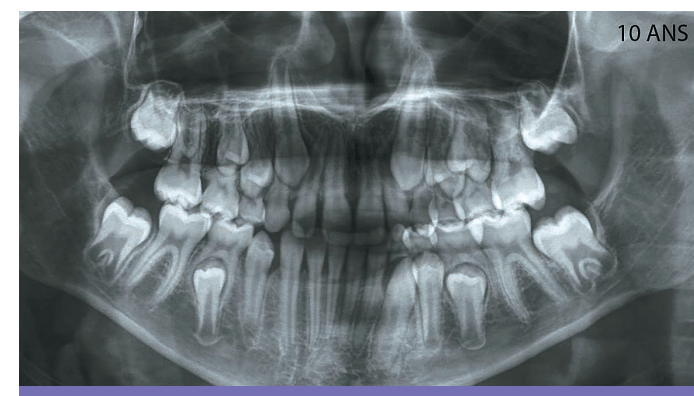

Fig. 9 a Inclusion de la canine permanente maxillaire droite (13) en l'absence d'intervention et de suivi à 13 ans. À l'âge de 9 ans, on peut observer la bascule de l'axe des canines maxillaires en cours d'évolution, 13 venant au contact de la face distale de l'incisive latérale permanente adjacente. dante peut faciliter l'éruption de la canine ectopique. Certaines conditions sont néanmoins nécessaires pour le succès du traitement : diagnostic précoce en denture mixte ; la position distomésiale radiologique de la pointe canine ne dépasse pas la moitié de la largeur de la racine de l'incisive latérale permanente adjacente; la couronne de la canine est plus basse que l'apex de l'incisive latérale permanente adjacente, l'angulation de la

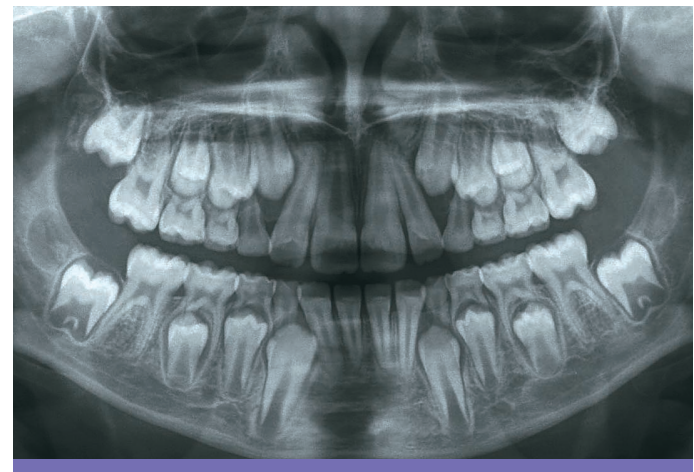

Fig. 8 Radiographie panoramique réalisée à l'âge de 10 ans suite à l'observation d'une rotation des incisives latérales. On observe la bascule de l'axe des canines permanentes maxillaires qui viennent au contact de la face distale des incisives latérales permanentes adjacentes.

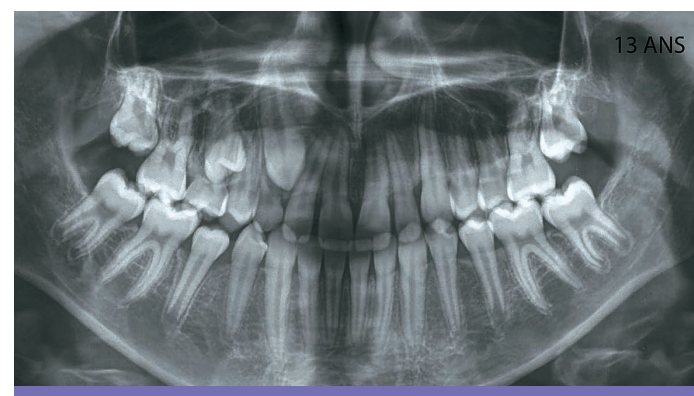

Fig. 9 b À 13 ans, les canines sont quasiment matures (fermeture radiographique des apex) et leur capacité d'évolution est limitée.

canine est inférieure à $30^{\circ}$; il existe un espace suffisant sur l'arcade pour l'évolution de la canine. Il est préférable de réaliser simultanément l'extraction de la canine controlatérale, de manière à minimiser le risque de dérive du point inter incisif. Un suivi régulier doit être mené afin de juger de l'évolution correcte des canines permanentes (fig. 9 a et b).

Dans les cas où il existe un encombrement important, il est possible de minimiser la perte 


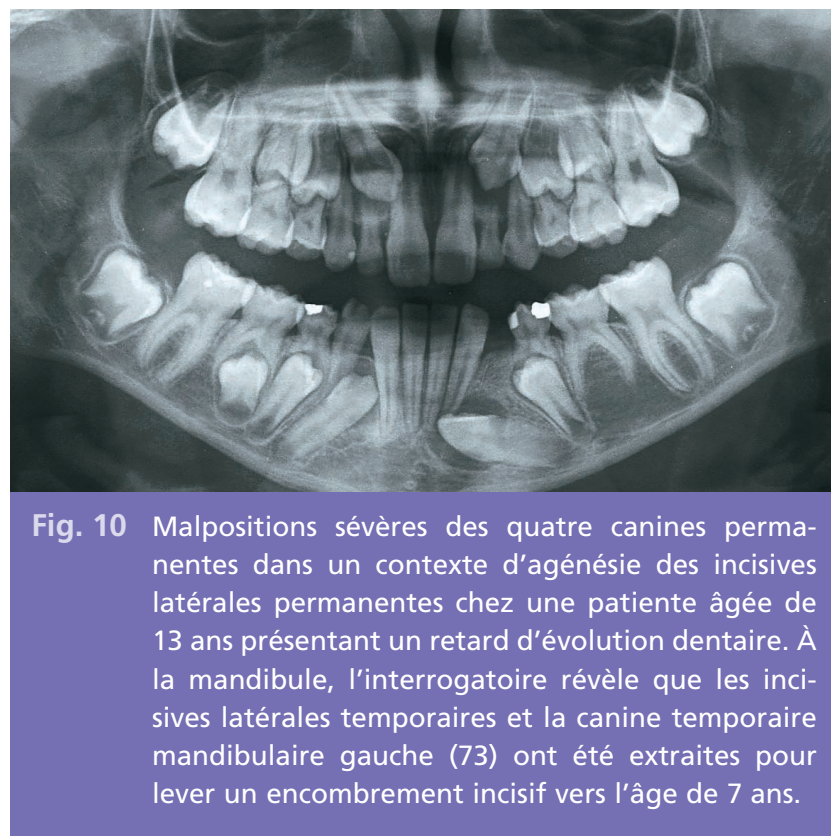

d'espace liée à l'extraction des canines temporaires par la pose simultanée d'un mainteneur d'espace[13].

Dans les cas de classe I sans dysharmonie dentomaxillaire ou présentant une dysharmonie dentomaxillaire modérée, quand les canines permanentes sont en voie d'inclusion ou en situation palatine ou vestibulaire d'éruption, I'extraction des canines temporaires correspondantes est le traitement préventif de choix chez les patients âgés de 10 à 13 ans[14, 15]. L'extraction de la canine temporaire permet alors de désenclaver la canine permanente dans $62 \%$ des cas, et d'améliorer sa position en vue d'une éventuelle traction orthodontique dans $17 \%$ des cas[16].

Néanmoins, si une résorption radiculaire des incisives latérales adjacente par les canines permanentes est visible avant cette tranche d'âge, les canines temporaires devront être extraites et un suivi clinique et radiographique régulier mis en place de façon à contrôler l'éruption de la canine.
Dans les cas de malposition sévère de la canine permanente, I'avis d'un spécialiste qualifié en orthopédie dentofaciale doit être recherché (fig. 10). En effet, l'extraction inutile de la canine temporaire nous privant alors de possibilité thérapeutique pour le futur, il est alors nécessaire d'évaluer la possibilité d'amener ces canines sur l'arcade par des moyens orthodontiques.

Temporaires ou permanentes, les canines possèdent un rôle clé dans l'organisation des arcades dentaires. Leur mise en place sur les arcades dentaires et leur conservation sont primordiales pour l'établissement et le maintien d'une occlusion et de fonctions orales correctes et pérennes. La détection de signes précoces et la prévention des troubles ainsi interceptés, souvent par des gestes simples permettent de prévenir les conséquences majeures que la dégradation de cette dent peut engendrer, tant en denture temporaire qu'en denture permanente. 


\section{Bibliographie}

1. Lautrou A.

Anatomie Dentaire.

$2^{\mathrm{e}}$ édition. Masson, Paris 1993;264 p.

2. Ravn JJ.

Occlusion in the primary dentition in three year old children.

Journal of the American Dental Association 1975;89:599-606.

3. Andrews LF.

The six keys of normal occlusion. American Journal of Orthodontics 1972,62:296-309.

4. Moyers RE. Handbook of orthodontics. $4^{\text {th }}$ edition Yearbook Medical Publishers, Inc. Chicago 1980.

5. Mathieu-Thonon G.

Bruxisme en denture temporaire.

Revue Belge de Médecine Dentaire 1969;24:113-122.

6. Deffez JP, Fellus $P$, Gerard CL. Rééducation de la déglutition salivaire. CdP, Paris 1995.

7. Fellus $P$. Orthodontie précoce en denture temporaire. CdP, Paris 2003.

8. Ovsenik M, Farcnik FM, Korpar M, Verdenik I.
Follow-Up study

of functional

and morphological

malocclusion trait

change from 3 to 12 years

of age.

European Journal

of Orthodontics

2007;29:523-529.

9. Jacobs SG.

The impacted

maxillary

canine.

Further observations

on aetiology,

radiographic localization, prevention/interception

of impaction,

and when to suspect

impaction.

Australian Dental Journal 1996;41:310-316.

10. Rayne J.

The unerupted maxillary canine.

The Dental Practitioner And Dental Record 1969;19:194-204.

11. Richardson $G$, Russell $K A$. A review of impacted permanent maxillary cuspids - diagnosis and prevention. Journal Of Canadian Dental Association 2000;66:497-501.

12. Shapira $Y$, Kuftinec $M N$.

Early diagnosis and interception of potential maxillary canine impaction. Journal Of American
Dental Association

1998;129:1450-1454.

13. Curzon MEJ, Roberts JF, Kennedy DB.

Kennedy's Paediatric Operative Dentistry. $4^{\text {th }}$ edition

Wright, London 1996.

14. Jacobs SG.

Reducing the incidences of palatally impacted maxillary canines by extraction of deciduous canines: a useful preventive/interceptive orthodontic procedure.

Case reports.

Australian Dental Journal 1992;37:6-11.

15. Rohlin $M$, Rundquist $L$.

Apical root anatomy of impacted maxillary canines.

A clinical and radiographic study. Oral Surgery

Oral Medecine

Oral Pathology 1984;58:141-147.

16. Power SM, Short MB. An investigation into the response of palatally displaced canines by extraction of deciduous canines and an assessment of factors contributing to favourable eruption. British Journal of Orthodontics 1993;20:217-223. 


\section{SUMMARY}

\section{Children's canine}

Mathieu A. DERBANNE

Marguerite-Marie LANDRU

\section{Keywords \\ - canine \\ - children \\ - growth \\ - deciduous teeth \\ - permanent teeth}

Position and functions of the deciduous and permanent canines closely intricate with growth and dentition phenomenon. By monitoring and correcting deciduous teeth position and function, the dental practitioner can prevent various dysmorphosis to appear in further dentition stages. Evolution of the permanent canine also represents a crucial moment in the evolution of the teeth. As for the deciduous canine, cautious and early monitoring can prevent serious troubles like dental inclusions of malpositions which might impair functions and esthetics of the teenager.

Canines, deciduous or permanent, much be subject to specific care at each step of their evolution and life: deciduous dentition, mixed dentition and permanent dentition. 